\title{
Hidrocefalia de pressão normal: visão atual sobre a fisiopatologia, diagnóstico e tratamento
}

\author{
Renan Muralho Pereira', Laura Mazeti', Deborah C. Pereira Lopes', Fernando Campos Gomes Pinto²
}

Faculdade de Medicina da Universidade de São Paulo (FMUSP).

\section{RESUMO}

A hidrocefalia de pressão normal (HPN), descrita em 1964 por Salomón Hakim, é uma doença insidiosa que acomete, sobretudo, idosos entre 60 e 80 anos e manifesta-se por meio da tríade clínica de distúrbios da marcha, demência e incontinência urinária, associada a achados radiológicos de ventriculomegalia e achados laboratoriais de pressão liquórica normal. Apesar de representar cerca de $5 \%$ das causas de demência, ficando atrás da doença de Alzheimer e demência vascular, ao contrário dessas causas a HPN é uma das poucas causas reversíveis de demência, e é por essa reversibilidade que torna fundamental o conhecimento da doença por parte de todos os médicos que cuidam dos pacientes dessa faixa etária. O artigo foi realizado a fim de apresentar as principais hipóteses relacionadas a fisiopatologia, diagnóstico e tratamento da HPN empregadas atualmente. A revisão da literatura foi realizada por meio das bases de dados Medline e PubMed, sendo utilizado o recurso MeSH (Medical Subject Headings) para a combinação dos descritores (hidrocefalia de pressão normal, história, epidemiologia, etiologia, fisiopatologia, diagnóstico e terapia) e seleção de artigos dos últimos seis anos. Tanto as bases fisiopatológicas quanto os critérios diagnósticos da HPN ainda não estão totalmente estabelecidos, porém as principais hipóteses envolvem distúrbios da hemodinâmica e complacência cerebral. O principal tratamento consiste na derivação liquórica, mediante a derivação ventriculoperitoneal e da terceira ventriculostomia endoscópica, sendo fundamental que o diagnóstico da HPN seja realizado precocemente e que os pacientes com maiores chances de responder à derivação liquórica sejam identificados.

\section{PALAVRAS-CHAVE}

Hidrocefalia de pressão normal, derivação ventriculoperitoneal, apraxia da marcha, demência, incontinência urinária.

\section{ABSTRACT}

Normal pressure hydrocephalus: current view on pathophysiology, diagnosis and treatment

The normal pressure hydrocephalus (NPH), described in 1964 by Salomón Hakim, is an insidious disease that affects mainly elderly people between 60 and 80 years and is manifested by the clinical triad of gait disturbances, dementia and urinary incontinence associated the radiological findings of ventriculomegaly and laboratory findings of normal cerebrospinal fluid pressure. Despite representing about $5 \%$ of the causes of dementia, behind Alzheimer's disease and vascular dementia, unlike these causes NPH is one of the few reversible causes of dementia, and it is this reversibility that makes crucial the knowledge of the disease by of all physicians who care of patients in this age group. The paper was developed in order to present the main hypotheses related to the pathophysiology, diagnosis and treatment of NPH in practice today. The literature review was conducted through Medline and PubMed, where was used the MeSH resource (Medical Subject Headings) for the combination of keywords (normal pressure hydrocephalus, history, epidemiology, etiology, pathophysiology, diagnosis and therapy and selection of articles from the last six years. Both the pathophysiology and diagnostic criteria of NPH has not been fully established, but the main hypotheses involve disturbances of cerebral hemodynamics and complacency. The main treatment consists of CSF shunt through the ventriculoperitoneal shunt and endoscopic third ventriculostomy and it's essential that the diagnosis of NPH be realized early and that patients with higher chance to improve after CSF shunt are identified.

\section{KEYWORDS}

Hydrocephalus, normal pressure, ventriculoperitoneal shunt, gait apraxia, dementia, urinary incontinence.

\section{Introdução}

\section{História da hidrocefalia de pressão normal}

A hidrocefalia de pressão normal (HPN) é uma síndrome neurológica caracterizada geralmente por meio de uma tríade de sintomas clínicos que consistem em apraxia de marcha, demência e incontinência urinária, associados à ventriculomegalia (detectada radiologicamente) e pressão normal do liquor cefalorraquidiano (LCR). ${ }^{1-3}$

Quem primeiro identificou essa síndrome foi o neurocirurgião Salomón Hakim, no Hospital San Juan de Dios, em Bogotá, Colômbia, em 1957, quando encontrou seu primeiro caso de HPN, a princípio

1. Acadêmico da Universidade Anhembi Morumbi e membro da Liga de Neurocirurgia da Faculdade de Medicina da Universidade de São Paulo (FMUSP), São Paulo, SP, Brasil.

2. Coordenador do Grupo de Hidrodinâmica Cerebral, Divisão de Neurocirurgia Funcional do Instituto de Psiquiatria, Hospital das Clínicas, Faculdade de Medicina, Universidade de São Paulo (IPq-HC-FMUSP), São Paulo, SP, Brasil. 
chamada de hidrocefalia sintomática oculta. O paciente era um menino de 16 anos de idade, vítima de um traumatismo cranioencefálico (TCE) resultante de um acidente automobilístico. Foi realizada a drenagem de um hematoma subdural no hemisfério direito, porém o paciente permaneceu em estado semicomatoso e foi diagnosticado como tendo danos cerebrais irreversíveis. Após a realização de uma pneumoencefalografia (Figura 1), foi identificada ventriculomegalia, porém a pressão intracraniana (PIC) apresentava-se normal $\left(150 \mathrm{mmH}_{2} \mathrm{O}\right.$ ), o que despertou o interesse de Hakim. ${ }^{1}$ Com objetivo de realizar análises laboratoriais, Hakim realizou uma punção lombar e retirou $15 \mathrm{ml}$ de LCR. De maneira inesperada, o paciente apresentou melhora no dia seguinte e, até mesmo, falou pela primeira vez, após meses sem falar. O paciente apresentou piora do quadro poucos dias depois, porém voltou a melhorar após uma nova drenagem lombar. A partir dessas observações, Hakim foi impelido a implantar uma derivação ventrículo-atrial, apesar da ausência de evidências comprovando sua eficácia em pacientes portadores de ventriculomegalia com PIC normal. Para sua surpresa, o paciente melhorou consideravelmente e retornou à escola apenas três meses após.

Esse caso foi relatado por Hakim em sua tese em 1964 e publicado no Journal of the Neurological Sciences em 1965, com outros dois casos de HPN cujos sintomas foram revertidos totalmente após a implantação de um sistema de derivação liquórica. ${ }^{1,2}$

As primeiras hipóteses que visaram à explicação dos mecanismos da hidrocefalia com PIC normal surgiram a partir do momento em que Salomón Hakim lançou mão de princípios da física, valendo-se da lei de Pascal para exemplificar e, sobretudo, reproduzir situações que lhe permitissem demonstrar como o aumento progressivo da área ventricular poderia instalar-se mesmo na ausência do aumento concomitante da pressão. ${ }^{3}$
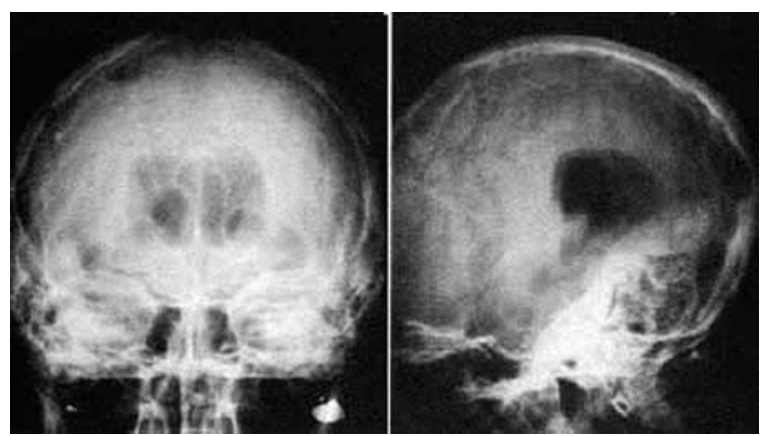

Figura 1 - Pneumoencefalografia do primeiro paciente de Salomón Hakim com HPN, que apresentou ventriculomegalia $e$, no entanto, PIC normal. Essas imagens foram publicadas em sua tese, intitulada: Some observations on C.S.F. pressure. Hydrocephalic syndrome in adults with "Normal" C.S.F. pressure [tese]. Universidade Javeriana da Faculdade de Medicina, Bogotá, Colômbia, S.A.,1964. p. $7 .{ }^{1}$
A lei de Pascal envolve as interações entre força, pressão e área, no interior de um sistema hidráulico, e Hakim utilizou esses princípios como base de seu raciocínio (Figura 2). De acordo com essa lei, quando duas colunas de água com áreas diferentes são submetidas a uma mesma pressão, a força exercida pela água na parede da coluna de maior área é maior do que na coluna de área menor, visto que a força é o produto da multiplicação entre pressão e área, como demonstrado pela equação (1a), em que F, P e A representam força, pressão e área, respectivamente. ${ }^{1,2} \operatorname{Logo}$, diante de uma mesma pressão, o aumento da área (equação $1 b$ ) resulta em aumento da força (equação 1c):

$$
\begin{aligned}
& F=P \times A \\
& F=P \times A \\
& F=P \times A
\end{aligned}
$$

Hakim demonstrou, mediante a utilização de bexigas elásticas em vários estágios de enchimento - cuja força elástica e pressão foram constantemente mensuradas -, que a pressão obtida tanto com bexigas totalmente infladas quanto parcialmente infladas poderia ser a mesma em alguns estágios de enchimento, porém, quando as forças elásticas de tais bexigas eram comparadas, as bexigas mais infladas apresentavam maior força elástica devido à maior área de superfície em que esta era aplicada (Figura 3). Após confirmar com seu experimento que pressões iguais poderiam coexistir com estágios de dilatação diferentes, Hakim associou seus conceitos prévios referentes às interações entre força, pressão e área aos seus resultados e, a partir disso, comparou seu sistema com o sistema ventricular e com a dinâmica liquórica (Figura 4). ${ }^{1,2}$

Dessa forma, Salomón Hakim concluiu que os pacientes apresentavam ventriculomegalia e PIC normal devido ao fato de que a existência de ventrículos alargados (aumento da área) resultava em uma

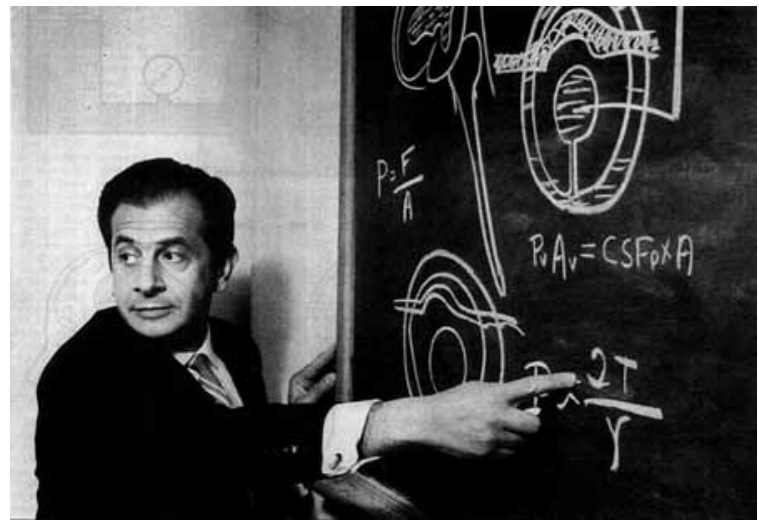

Figura 2 - Dr. Salomón Hakim descrevendo as propriedades físicas da HPN envolvendo pressão, força e área. Essa fotografia encontra-se no artigo de Wallensteins MB, McKhann GM. Salomón Hakim and the discovery of normal-pressure hydrocephalus. Neurosurgery. 2010;67(1):155. ${ }^{4}$ 
neutralização relativa dos aumentos pressóricos. No entanto, percebeu que nessas condições a força exercida nas paredes ventriculares era consideravelmente elevada e que era esse aumento o causador da lesão hidráulica cerebral e, consequentemente, das mani- festações clínicas da doença. ${ }^{1,4} \mathrm{~A}$ partir dos estudos de Hakim, grandes esforços foram feitos a fim de melhor caracterizar e diagnosticar a HPN, bem como prever a probabilidade de melhora após o implante de um sistema de derivação liquórica. ${ }^{5-11}$
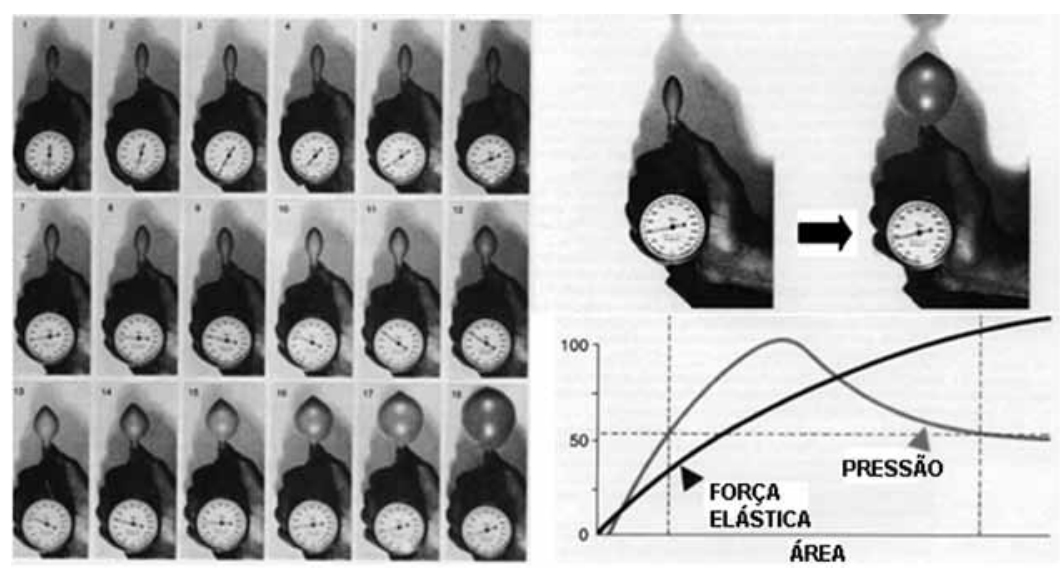

Figura 3 - Demonstração do efeito da prensa hidráulica, por meio da aplicação da lei de Pascal. Hakim demonstrou que a pressão permanece constante em estágios diferentes de enchimento das bexigas (ponta de seta cinza). No entanto, a força elástica no interior das bexigas mais infladas era maior do que em bexigas menos infladas (ponta de seta preta). Dessa forma, conseguiu comprovar como era possível a coexistência entre áreas diferentes e pressões semelhantes no interior de um sistema hidráulico. Essas imagens foram publicadas na tese de Hakim em 1964, intitulada Some observations on C.S.F. pressure. Hydrocephalic syndrome in adults with "Normal" C.S.F. pressure [tese]. Universidade Javeriana da Faculdade de Medicina, Bogotá, Colômbia, S.A., 1964. p. 30-3. ${ }^{1}$ (Modificada)

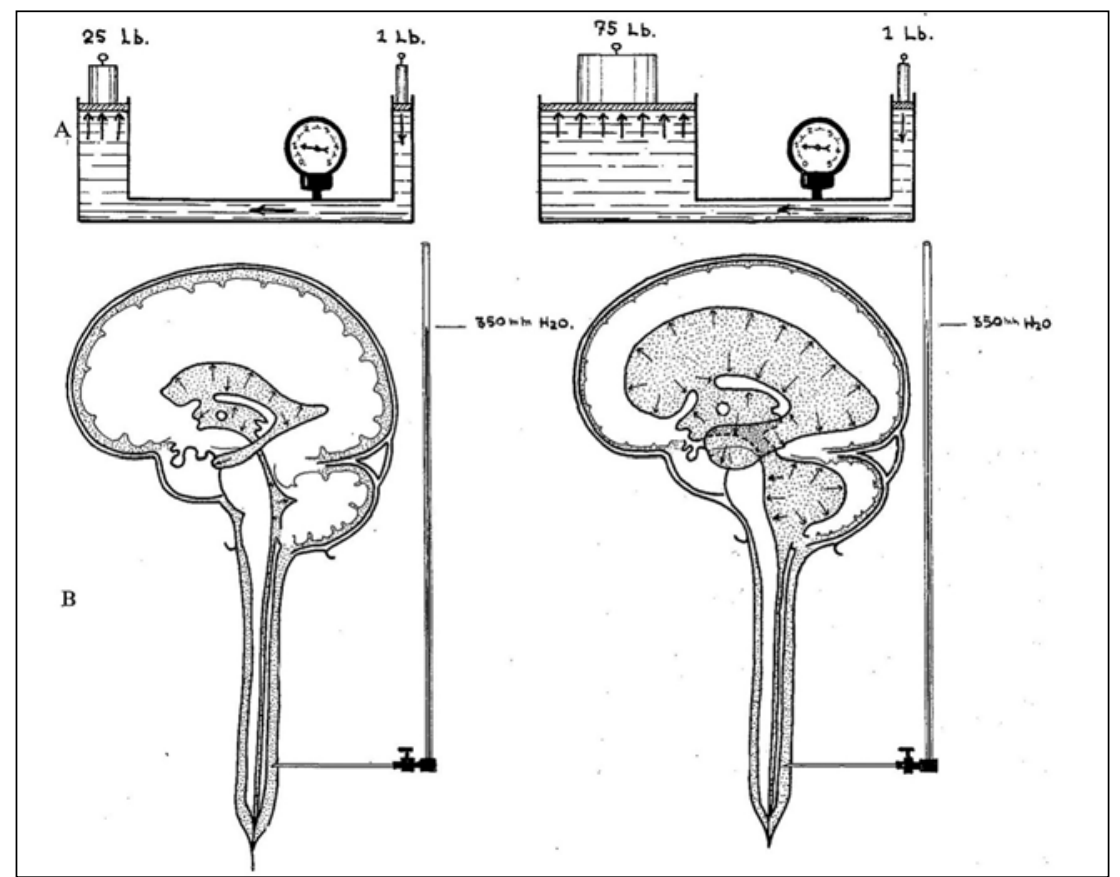

Figura 4 - Comparação entre o sistema ventricular e as propriedades hidrodinâmicas da lei de Pascal. A comparação demonstra que tanto em sistemas hidráulicos quanto em sistemas ventriculares que possuem áreas diferentes, sob a mesma pressão, os sistemas de área maior exercem forças maiores sobre suas paredes. Dessa forma, Hakim atribui as lesões neurológicas da HPN a esse aumento de força exercida sobre neurônios periventriculares. Esta imagem foi publicada no artigo de Hakim S, Adams $R D$. The especial clinical problem of symptomatic hydrocephalus with normal cerebrospinal fluid pressure. J Neurol Sci. 1965;2(4):325-7.2 


\section{Epidemiologia}

A incidência e a prevalência da HPN ainda não são precisas e muitos estudos relatam valores diferentes. Tal fato se deve grande parte à ausência de um consenso universal quanto às etapas referentes ao diagnóstico da doença, que resulta tanto em subdiagnósticos quanto em diagnósticos errados, visto que muitas vezes a HPN idiopática (HPNi) pode ser "confundida" com outras etiologias de apresentação semelhante, sobretudo em seus estágios iniciais e quando as características da tríade não se manifestam ao mesmo tempo.

Brean e Eide ${ }^{5}$, em estudo que realizou uma busca ativa, por meio de extensa campanha de mídia, em uma população norueguesa de 220.000 habitantes atendidos por um único centro neurológico, a fim de recrutar possíveis casos de $\mathrm{HPN}$, relataram incidência de HPNi de 5.5/100.000 e prevalência de 21.9/100.000 habitantes. Além disso, quando considerados apenas pacientes com idade acima de 65 anos, a prevalência foi de 117,9 por 100.000 habitantes.

A HPN pode ser dividida em duas categorias: secundária e idiopática. A HPN secundária ocorre em seguida a eventos neurológicos de base, como hemorragia subaracnóidea (HSA) e hemorragia intraventricular causada por traumas ou ruptura de aneurismas e meningites. Em contrapartida, a HPNi ocorre geralmente entre a sexta e a oitava década de vida e ainda não tem seus mecanismos fisiopatológicos completamente definidos. ${ }^{12}$ Neste trabalho daremos prioridade para a HPNi.

\section{Manifestações clínicas}

A HPN manifesta-se, em geral, por meio da tríade clínica composta por apraxia de marcha, demência e incontinência urinária combinada com achados radiográficos de ventriculomegalia e laboratoriais de pressão normal do (LCR). ${ }^{9,12}$ Os sinais e sintomas tipicamente se desenvolvem de forma insidiosa, bilateralmente, mas podem aparecer lateralizados quando superpostos por condições coexistentes, como acidente vascular encefálico (AVE), radiculopatia e neuropatia periférica e geralmente ocorrem entre a sexta e a oitava década de vida. ${ }^{13,14}$

A apresentação clínica da HPN pode, ainda assim, variar significativamente quanto à gravidade e à progressão desses sintomas, portanto não é necessário que toda a tríade esteja presente para ser considerado o diagnóstico de $\mathrm{HPN}^{7}$ (Tabela 1).

Quanto aos elementos da tríade, a alteração de marcha usualmente aparece antes da demência e incontinência urinária, sendo na maioria dos casos o sinal clínico mais proeminente nos estágios iniciais da doença. ${ }^{12}$

Em virtude de a HPN ser uma doença, sobretudo, da população idosa, faixa etária em que a dificuldade de marcha, demência e incontinência urinária são relativamente comuns, uma diversidade de diagnósticos de sintomas individuais deve ser considerada, incluindo doenças neurodegenerativas, etiologias vasculares e desordens urológicas. Uma relação de diagnósticos diferenciais da HPN é apresentada na tabela 1 e, e diante de tais diagnósticos, torna-se evidente a importância de uma avaliação clínica adequada, tanto por parte dos clínicos-gerais quanto por parte dos geriatras e, finalmente, dos neurologistas que estiverem assistindo os pacientes que apresentarem características da HPN, visto que o diagnóstico precoce e, sobretudo, correto guarda estreita relação com o sucesso terapêutico. ${ }^{7,9,12,15}$

No ano de 2005, foi publicado como suplemento do periódico Neurosurgery um extenso trabalho denominado "Guidelines for the diagnosis and management of idiopathic NPH”. ${ }^{23}$ Essa diretriz aborda aspectos fundamentais - referentes ao reconhecimento, diagnóstico, utilização de exames suplementares e tratamento cirúrgico - da HPNi, que abordaremos ao longo deste artigo.

Tabela 1 - Diagnósticos diferenciais da $\mathrm{HPN}^{15}$

\begin{tabular}{lll} 
& & \multicolumn{2}{c}{ Distúrbios } \\
Neurodegenerativas & Demência vascular & Outras causas \\
\hline Doença de Alzheimer & Infartos múltiplos & Hidrocefalia obstrutiva \\
Doença de Parkinson & Doença de pequenos & Hidrocefalia congênita \\
Corpos de Lewy & vasos cerebrais & Tumor na coluna espinal \\
Doença de Huntington & Doença de Binswanger & Deficiência de vitamina B12 \\
Demência frontotemporal & Acidente vascular cerebral & Estenose espinal \\
Atrofia de múltiplos sistemas & Insuficiência & Lesão cerebral traumática \\
Encefalopatia espongiforme & vértebro-basilar & \\
Degeneração corticobasal & & \\
\hline Estas doenças podem manifestar-se com sinais e sintomas semelhantes ao da HPN. Logo, muitos pacientes podem ser diagnosticados de forma errônea. Portanto, \\
é imprescindível que se realize uma avaliação clínica e neurológica adequada, a fim de diferenciar a HPN dessas doenças e instituir o tratamento correto.
\end{tabular}




\section{Alteração de marcha}

Geralmente, o distúrbio de marcha e algum outro sintoma são necessários para realizar o diagnóstico, tendendo a ser a característica mais reconhecível da HPN. ${ }^{4,15}$ Embora nenhuma característica seja patognomônica da alteração de marcha na HPN, esta se apresenta como uma marcha lenta, de base alargada, passos curtos com os pés arrastados pelo chão (como uma "marcha magnética"), dificuldade de virar-se, realizar o teste do "pé ante pé" e iniciar os primeiros passos. Não há fraqueza motora e os pacientes frequentemente apresentam história de quedas. Desequilíbrio e diminuição da velocidade da marcha (por causa dos passos curtos e apraxia da marcha) também são comuns. ${ }^{15}$ Entretanto, o balanço dos braços ao andar encontra-se preservado, o que pode ajudar na distinção entre a marcha da doença de Parkinson. ${ }^{16}$

A alteração de marcha pode surgir como uma dificuldade em subir ou descer escadas e andar no ritmo esperado. Os pacientes podem se queixar de dificuldade para levantar de uma cadeira, fraqueza das extremidades inferiores e fadiga ao andar. Além disso, muitas vezes mostram inabilidade para subir na mesa de exame ou em suas próprias camas. ${ }^{9,15}$

A origem da alteração de marcha não é totalmente compreendida, porém algumas hipóteses foram sugeridas. Umas das hipóteses sugere que o aumento dos ventrículos na HPN causa uma compressão e/ou deformação das fibras dos neurônios motores superiores que passam pela porção medial da corona radiata. ${ }^{12,17}$

Outras condições que causam anormalidade de marcha devem ser consideradas ao realizar o diagnóstico da HPN como estenose de canal lombar, neuropatia periférica, doença cerebrovascular, doença de Alzheimer (DA), tumores, trauma, doença de Parkinson, demência dos corpos de Lewy, depressão, alcoolismo crônico, deficiência de vitamina B12 e ácido fólico. ${ }^{12,18}$ Além disso, a anormalidade de marcha é o sinal mais responsivo ao tratamento com a implantação de um sistema de derivação liquórica. ${ }^{12,14,19,20}$

\section{Alterações cognitivas}

A alteração cognitiva geralmente á a segunda manifestação da HPN após o distúrbio de marcha. É caracterizada por um progressivo déficit das funções das áreas subcortical e frontal, manifestado como retardo psicomotor, diminuição da atenção e concentração, disfunção executiva e visoespacial e alteração de memória, sendo a função executiva danificada precocemente no curso da doença. Os déficits da área cortical como apraxia, agnosia e afasia são raros. ${ }^{7}$ A severidade de tais déficits vistos na HPN pode variar de minimamente detectáveis a profundamente severos ${ }^{17}$, e significativa melhora desses sintomas pode ser obtida depois da derivação, sendo, portanto, uma das poucas causas de demência reversível. Uma breve avaliação pode ajudar a determinar a causa do prejuízo da cognição. $\mathrm{O}$ Miniexame do Estado Mental (Mini Mental) pode mostrar déficits para realização de cálculos, concentração, escrita de frases, copiar a intersecção de pentágonos e seguir um comando de três estágios. Processamento mental lentificado pode ser evidente por causa do aumento do tempo do teste. O desempenho no teste do relógio também pode estar alterado, mostrando um pobre planejamento espacial e organização. ${ }^{21}$ Distúrbios de comportamento também foram relatados associados à HPN como depressão, mania, agressividade, distúrbio obsessivo-compulsivo, psicoses incluindo paranoia, alucinações e distúrbios do controle do impulso. $\mathrm{O}$ aparecimento de sintomas depressivos em pacientes com HPN pode ser uma consequência neuroquímica da desordem cerebral. Entretanto, pode aparecer em resposta à incapacidade física e mental associada à HPN. O local de disfunção responsável pela demência na HPN permanece incerto, embora o sistema frontoestriatal tenha sido implicado por alguns investigadores. Outros enfatizam a importância de outras estruturas subcorticais, incluindo fibras de projeção passando próximo aos ventrículos laterais. ${ }^{9,17}$

O diagnóstico diferencial pode ser particularmente difícil por causa da similaridade das alterações cognitivas com doenças mais prevalentes como a DA. Geralmente, a DA está mais associada com alteração de memória, aprendizado e orientação, enquanto a HPN mais frequentemente está associada com diminuição psicomotora e da velocidade motora fina. As duas doenças podem levar a uma perda de função executiva, atenção e concentração, mas a HPN dificilmente causa alterações precoces de linguagem. A existência da DA e HPN concomitantemente não é incomum, e a probabilidade de cada uma aumenta com a presença de idade avançada e hipertensão arterial sistêmica (HAS). ${ }^{22} \mathrm{Em}$ casos coexistentes de HPN e DA, atrofia hipocampal pode ser observada na ressonância magnética $(\mathrm{RM}) .{ }^{18}$

\section{Incontinência urinária}

A incontinência urinária normalmente é observada nos estágios avançados da doença e parece ocorrer por uma interrupção das vias neuronais periventriculares que se direcionam ao centro sacral da bexiga urinária, levando à hiperatividade do músculo detrusor, que pode 
resultar primariamente em urgência urinária e polaciúria. ${ }^{12,21}$ Pode ocorrer, também, devido ao distúrbio da marcha, apraxia e bradicinesia que impedem o paciente de chegar ao banheiro a tempo e, em casos extremamente avançados, pode resultar de falta de preocupação para micturição, sugerindo que a incontinência é associada com uma disfunção executiva frontal., ${ }^{9} 12$

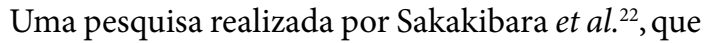
avaliou 42 pacientes com possível HPN, submetidos à exame de urodinâmica, revelou que $95 \%$ dos pacientes apresentaram evidência de hiperatividade do músculo detrusor, 93\% apresentaram sintomas do trato urinário inferior, e tais sintomas incluíam sintomas de armazenamento em $93 \%$ dos pacientes e sintomas de esvaziamento em $71 \%$ dos pacientes. Tal estudo demonstrou que em estágios iniciais a urgência urinária pode anteceder a incontinência nos pacientes com HPN.

A incontinência não é um componente invariável da doença e pode ocorrer raramente ou apenas intermitentemente em alguns casos. A polaciúria apresenta tipicamente mais de seis episódios em 12 horas, sendo a noctúria comum. ${ }^{7} \mathrm{~A}$ incontinência também pode ser causada por doenças que afetam o controle autonômico da bexiga, como a neuropatia diabética. Outras causas frequentes são o uso de diuréticos e fármacos com efeito anticolinérgico e perda multifatorial do controle da bexiga como aumento da próstata e prolapso de órgãos pélvicos. ${ }^{18,23}$

\section{Fisiopatologia}

Apesar de ter sido descrita há quase 50 anos, a fisiopatologia da HPN ainda permanece não totalmente definida. Grandes esforços foram feitos na tentativa de elucidar seus mecanismos de instalação e tais esforços originaram diversas hipóteses causais que têm servido de base para sua explicação atualmente. Tais hipóteses variam amplamente entre si e incluem absorção liquórica deficiente, isquemia da substância branca profunda, redistribuição das pulsações vasculares e diminuição da complacência do parênquima cerebral, vasos sanguíneos e espaço subaracnóideo. ${ }^{11,14,24} \mathrm{Em}$ seguida descreveremos as principais hipóteses relacionadas à fisiopatologia da HPN.

Salomón Hakim descreveu o aumento da área ventricular como um requisito muito importante na HPN e afirmou que é devido a esse aumento da área que se desenvolve uma força prejudicial aos circuitos neuronais periventriculares, mesmo na presença de PIC normal. ${ }^{1-3}$ Apesar de essa descrição ser um bom "ponto de partida" em relação à tentativa de explicar a fisiopatologia da
HPN, Hakim não esclareceu os motivos pelos quais o sistema ventricular torna-se aumentado, predispondo ao aumento da força exercida sobre suas paredes. A fim de responder a tal questão, originaram-se diversas hipóteses, sobretudo referentes à hemodinâmica cerebral e à dinâmica liquórica. ${ }^{3,11,14}$

Alguns autores associam a hipoperfusão cerebral ao desenvolvimento da HPN. ${ }^{6,11,24}$ Tal associação se deve, principalmente, à importante influência do ciclo cardíaco sobre a dinâmica liquórica. Durante a sístole ventricular ocorre aumento do espaço ocupado pelo parênquima cerebral, devido à elevação do influxo sanguíneo intracraniano proveniente dos sistemas carotídeos e vértebro-basilar, gerando picos sistólicos intracranianos. Esse aumento de espaço requerido pelo parênquima cerebral resulta em expansão cerebral que provoca uma contração ventricular, devido à sua menor resistência. Essa contração ventricular causa ejeção de pequena quantidade de LCR ao longo de todo o sistema ventricular (dos ventrículos laterais para o terceiro ventrículo, deste para o quarto ventrículo, passando pelo aqueduto do mesencéfalo e finalmente para o espaço subaracnóideo). Um exemplo da influência do ciclo cardíaco sobre a dinâmica liquórica é demonstrado na figura 5. Além disso, outra pequena quantidade de LCR entra no interior do canal espinhal, que atua como um reservatório durante a sístole. ${ }^{13}$ Durante a diástole, o volume cerebral diminui, uma vez que ocorrem um efluxo do sangue venoso e diminuição do influxo arterial. Essas alterações permitem a reentrada do LCR do canal espinhal no crânio, resultando em fluxo retrógrado (caudocranial) de LCR dentro do sistema ventricular. ${ }^{13}$ Como demonstrado na figura 5 , a força compressiva originada pela sístole deve ser progressiva e contínua ao longo do sistema ventricular, garantindo que haja vazão adequada de LCR pelo aqueduto do mesencéfalo. Para que a dinâmica liquórica seja sempre unidirecional durante a sístole, o parênquima cerebral deve estar saudável e o sistema arterial deve estar íntegro bilateralmente, visto que a "força motriz" responsável pelo fluxo do LCR é a pulsação arterial. O comprometimento da "simetria sistólica" cerebral, causada por doenças como hipertensão arterial, aterosclerose e isquemia cerebral, pode resultar em um fluxo liquórico alterado e multidirecional, causando estase do LCR e, principalmente, impactação dele sobre as paredes ventriculares durante os picos sistólicos. Esse impacto liquórico causa tanto lesão mecânica (compressão do parênquima, capilares, vênulas e veias corticais superficiais) quanto tóxica/osmótica (diminuição do clearance de resíduos metabólicos e lesão ependimária, que resultam em aumento da carga osmótica), levando à dilatação ventricular e à lesão neuronal ainda maior, respectivamente (Figura 6). ${ }^{13}$ 
a

b

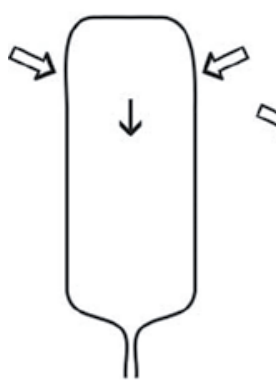

C

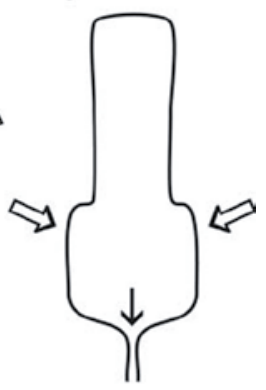

Figura 5 - Desenho simplificado do terceiro ventrículo e aqueduto do mesencéfalo. As setas maiores indicam as forças de compressão agindo de maneira coordenada sobre o ventrículo durante a sístole. As setas sólidas indicam o fluxo sistólico unidirecional de LCR resultando em vazão adequada dele. Situação normal. a-c: início, meio e final da sístole. Esse desenho foi publicado no artigo de Chrysikopoulos $H$. Idiopathic normal pressure hydrocephalus: thoughts on etiology and pathophysiology. Med Hypotheses. 2009;73(5):718-24. ${ }^{13}$
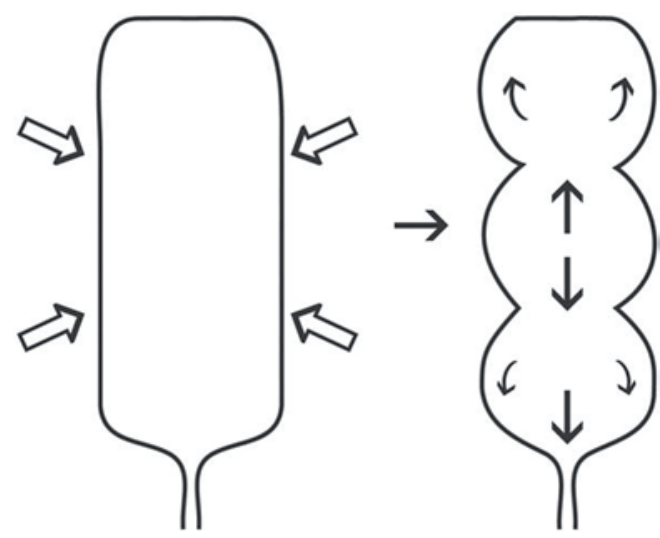

Figura 6 - Desenho simplificado do terceiro ventrículo em um plano transaxial com o aqueduto do mesencéfalo na regial inferior. As setas maiores demonstram a influência da sístole sobre a compressão ventricular. As setas sólidas demonstram o fluxo de LCR. A compressão inadequada simultânea (proximal e distal) do ventrículo resulta em compartimentalização ventricular, acúmulo de pressão e vazão diminuída de LCR. Esse desenho foi publicado no artigo de Chrysikopoulos $\mathrm{H}$. Idiopathic normal pressure hydrocephalus: thoughts on etiology and pathophysiology. Med Hypotheses. 2009;73(5):718-24. ${ }^{13}$

Takeuchi et al. ${ }^{11}$ avaliaram 44 pacientes com possível HPNi. Desses, 40 foram submetidos a algum sistema de derivação liquórica [derivação ventriculoperitoneal (DVP) e derivação lomboperitonial (DLP)], e seu fluxo sanguíneo cerebral regional cortical e profundo (tálamo e núcleos da base) foi mensurado um mês antes e após a cirurgia. Os 40 pacientes apresentaram fluxo sanguíneo cerebral diminuído antes da cirurgia e observou-se relação positiva entre o aumento do fluxo sanguíneo cerebral médio após a implantação da derivação (de $32.1 \pm 2.74$ $\mathrm{ml} / 100 \mathrm{~g} / \mathrm{min}$ antes da cirurgia para $39.8 \pm 3.02 \mathrm{ml} / 100 \mathrm{~g} /$ min após a cirurgia) e a eficácia do sistema de derivação.

Takaya et al. ${ }^{25}$ avaliaram o fluxo sanguíneo cerebral em 35 áreas por meio da tomografia computadorizada com emissão de fóton único (SPECT) de 21 pacientes: sete com sintomas objetivos da tríade da HPN, sete sem sinais objetivos e sete controles. Observaram que os pacientes sem sinais objetivos apresentavam hipoperfusão global comparada com o grupo controle. Tal hipoperfusão era semelhante à dos pacientes com os sinais objetivos da tríade, exceto na substância branca frontal, onde não apresentavam hipoperfusão. Portanto, concluíram que outros fatores além da hipoperfusão cerebral são responsáveis pela fisiopatologia da HPN, visto que os pacientes sem sinais objetivos também já apresentavam hipoperfusão global, assim como os pacientes em estágio clínico.

Bateman ${ }^{24}$ também discorda da hipótese da hipoperfusão cerebral como base fisiopatológica da HPN. Ele selecionou 20 pacientes com diagnóstico de HPN e fluxo sanguíneo cerebral acima do normal (> $700 \mathrm{ml} /$ min), que foram submetidos a exames de RM que avaliaram os seguintes itens: fluxo venoso no seio sagital superior (SSS) e seio reto (SR); influxo arterial total e o volume médio de LCR que passava pelo aqueduto do mesencéfalo em direção ao quarto e terceiro ventrículos, durante a sístole e diástole, respectivamente (aqueduct stroke volume). Os pacientes foram comparados com 12 controles. Observou-se fluxo sanguíneo venoso profundo (SR) normal e fluxo sanguíneo superficial (SSS) diminuído (com efluxo diminuído em $9 \%$ comparado com o influxo e complacência venosa diminuída em $50 \%$ ). Dessa forma, o autor afirma que a HPN pode ocorrer independentemente da perfusão arterial e fluxo venoso profundo e reconhece a diminuição do fluxo venoso superficial como potencial causador da HPN.

\section{Diagnóstico}

O diagnóstico da HPN requer evidências convergentes da história clínica, exame físico e estudos de neuroimagem, que incluem tomografia computadorizada (TC) e RM. A RM é superior à TC. A TC pode excluir a hipótese da HPN e é útil para a triagem inicial, enquanto a RM fornece informações mais precisas e permite a identificação de anormalidades estruturais e a causa da HPN. Além disso, a RM pode ser utilizada para a obtenção de informações fisiológicas adicionais, como a estimativa do fluxo de CSF por meio de imagens ponderadas em T2. ${ }^{12} \mathrm{~A}$ HPN se manifesta tipicamente entre 60 e 80 anos de idade, como uma doença progressiva, crônica e que não possui antecedente de causa. Alteração de marcha e equilíbrio são sintomas comuns e podem incluir distúrbios na cognição e controle uri- 
nário. A presença de aumento ventricular (índice de Evans $\geq 0,3$ ) nos exames de neuroimagem é necessária, porém não é suficiente para estabelecer o diagnóstico de HPN. Os resultados da neuroimagem (Figura 7) necessitam de interpretação associada aos dados da história clínica e achados físicos para que o diagnóstico correto da HPN seja realizado e para que se possa diferenciá-la de outras doenças. ${ }^{9,15}$

A HPN pode ser similar ou ocorrer em associação com várias doenças que são prevalentes nos idosos, como doença cerebrovascular, desordens neurodegenerativas, desordens urinárias primárias, estenose espinal e outras condições. ${ }^{15}$ A diretriz de 2005 sobre HPNi fornece critérios para o diagnóstico clínico da HPN, dividindo-o em três categorias: provável, possível e improvável. Essa classificação pode ser feita, frequentemente, na primeira visita clínica e leva em consideração a história, neuroimagem, clínica e testes fisiológicos do paciente. Para ser classificada em provável, a história do paciente deve incluir: acometimento insidioso; origem após os 40 anos; duração mínima de três a seis meses; nenhuma evidência de antecedentes como trauma de cabeça, hemorragia intracerebral, meningite ou outra condição conhecida de hidrocefalia secundária; progressão no tempo e nenhuma outra condição neurológica/psiquiátrica que seja suficiente para explicar a presença dos sintomas. Exames de neuroimagem, realizados após o início dos sintomas, devem mostrar evidência de aumento ventricular não inteiramente atribuído a atrofia cerebral ou aumento congênito (índice de Evans $\geq 0,3$ ); nenhuma obstrução macroscópica do fluxo liquórico e pelo menos uma das

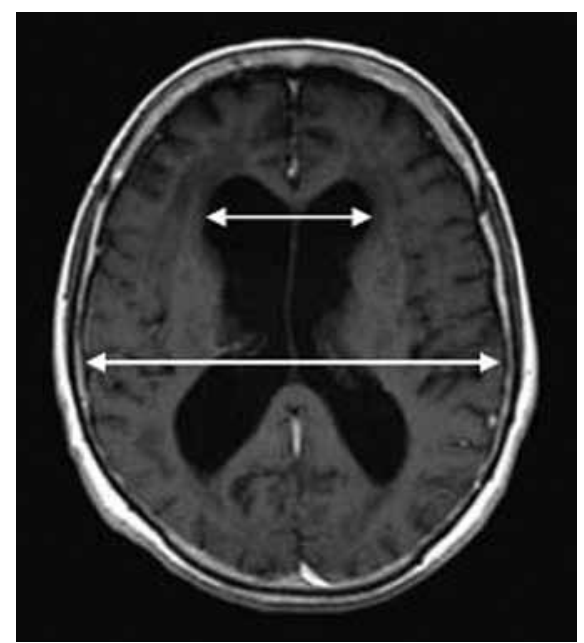

Figura 7 - Imagem de ressonância magnética mostrando ventriculomegalia em um paciente com HPN. O índice de Evans é a largura máxima dos cornos anteriores (seta menor) dividida pela largura máxima da calvária (seta maior). Hidrocefalia é definida por um índice maior ou igual a 0,3. O índice desse paciente é de 0,4. Essa figura foi publicada no artigo de Factora

$R$. When do common symptoms indicate normal pressure hydrocephalus? Cleve Clin J Med. 2006;73(5):452. ${ }^{18}$ características de suporte como aumento dos cornos temporais dos ventrículos laterais, não inteiramente atribuído à atrofia hipocampal; ângulo caloso igual ou maior que 40 graus; evidência de alteração no conteúdo de água no cérebro, incluindo alterações do sinal periventricular na TC e RM que não seja atribuída a alterações microvasculares isquêmicas ou de desmielinização e alteração do fluxo aquedutal ou do quarto ventrículo. Na clínica, achados de distúrbio de marcha devem estar presentes, pelo menos uma área de alteração na cognição e sintomas urinários, ou ambos. Com respeito ao distúrbio de marcha, pelo menos dois dos seguintes devem estar presentes (e não ser totalmente atribuídos a outras condições): altura do passo diminuída, comprimento do passo diminuído, ritmo (velocidade da marcha) diminuído, balanço do tronco aumentado, base alargada, dedos dos pés virados para fora ao andar, retropulsão (espontânea ou provocada), virar-se em bloco (virar-se requer três ou mais passos para realizar volta de 180 graus) e equilíbrio alterado ao andar, evidenciado por duas ou mais correções em oito passos do teste do "pé ante pé". Em relação à cognição, deve-se ter uma alteração documentada (ajustada à idade e educação) e/ou diminuição do desempenho em um teste cognitivo (como o Minimental) ou evidência de pelo menos dois dos seguintes no exame, que não sejam totalmente atribuídos a outras condições, como diminuição do desempenho psicomotor (aumento da latência de resposta); velocidade motora fina diminuída; precisão motora fina diminuída; dificuldade de dividir ou manter atenção; alteração de memória, principalmente para eventos recentes; disfunção executiva, como dificuldade em procedimentos de múltiplos passos, memória de trabalho, formulação de abstrações/similaridades, percepção e alterações de personalidade e comportamento. Para documentar os sintomas relacionados à incontinência urinária, um dos seguintes sintomas deve estar presente: incontinência urinária episódica ou persistente, não atribuída às doenças urológicas primárias; incontinência urinária persistente e incontinência urinária e fecal; ou dois dos seguintes devem estar presentes: urgência urinária (definida como percepção frequente de uma necessidade de urinar); frequência urinária (definida como mais de seis episódios de micção em uma média de 12 horas, apesar do volume normal) e noctúria (definido como a necessidade de urinar mais de duas vezes, em média, por noite).$^{15}$

Quanto à avaliação fisiológica, a pressão de abertura do LCR, determinada por punção lombar ou um procedimento comparável, deve estar em uma faixa de 105-190 mm H2O - medidas apropriadas da pressão maiores ou menores que essa faixa não são consistentes com o diagnóstico de HPNi provável.

Para ser classificada como possível, a história pode conter sintomas como: início recente ou indeterminado; 
começo em qualquer idade depois da infância; duração de menos três meses ou indeterminada; seguir eventos como trauma de cabeça, história remota de hemorragia intracerebral, meningite na infância ou na adolescência ou outras condições; coexistir com outra condição neurológica, psiquiátrica ou doenças gerais, mas que, na avaliação do clínico, não seja inteiramente atribuída a essas condições e que não seja progressiva ou claramente progressiva. A neuroimagem apresentando aumento dos ventrículos, mas associada à evidência de atrofia cerebral, com severidade suficiente para explicar o tamanho do ventrículo e lesões estruturais que podem influenciar no tamanho do ventrículo. Sintomas de incontinência e/ou alteração cognitiva, na ausência de distúrbio de marcha ou equilíbrio. Avaliação fisiológica da pressão de abertura não disponível ou resultados nos extremos da taxa de variação esperada (60-104 ou 191-240 mm $\mathrm{H} 2 \mathrm{O}$ ) são condizentes com HPNi possível. ${ }^{6,713}$

Finalmente, a HPNi é considerada improvável quando: não há evidência de ventriculomegalia; há sinais de aumento da pressão intracraniana como o papiledema; nenhum componente da tríade clínica da HPN está presente, os valores da pressão de abertura do LCR encontram-se fora das variações da HPNi "possível" (< 60 ou $>240 \mathrm{~mm} \mathrm{H}_{2} \mathrm{O}$ ) e quando os sintomas são explicados por outras causas. Ao elucidar a história, o clínico deve dar atenção ao modo como os sintomas iniciaram (agudo e subagudo) seu curso temporal (estático, progressivo) e sua severidade (leve, moderada, severa). Deve-se dar ênfase, especialmente, aos sintomas envolvendo marcha, equilíbrio, cognição e incontinência urinária. Ocorrência familiar de HPN é observada raramente (em contraste com a hidrocefalia congênita). Porém, é recomendado que elementos da história familiar sejam obtidos, com ênfase em doenças neurodegenerativas como doença de Parkinson, DA e doença de Huntington, assim como outras condições neurológicas e psiquiátricas que tenham caráter hereditário e possam assemelhar-se com a HPN..$^{15}$

Apesar da importância da avaliação clínica para a realização do diagnóstico de $\mathrm{HPNi}$, o grau de certeza fornecido apenas pelo diagnóstico clínico, relacionado à melhora do paciente após a implantação de um sistema de derivação liquórica, varia de menos de $50 \%$ até $60 \% .{ }^{26}$ Portanto, visto ser tal cirurgia um procedimento invasivo que pode acarretar complicações, especialmente em pacientes de idade avançada, como os portadores da HPN, torna-se necessária a utilização de testes suplementares com duas finalidades: (1) confirmar o diagnóstico e (2) identificar quais pacientes mais provavelmente melhorarão após a intervenção cirúrgica e predizer com maior acurácia a probabilidade dessa melhora. ${ }^{7,12,24}$ Os testes suplementares mais utilizados são os seguintes:

- Teste de punção lombar (tap test): é realizada uma punção lombar e removidos cerca de 40-
$50 \mathrm{ml}$ de LCR. O paciente é observado quanto às alterações na marcha e/ou à função cognitiva cerca de 30 a 60 minutos depois. Uma resposta positiva ao teste tem um grau de certeza maior para uma resposta favorável à colocação de uma derivação liquórica do que pode ser obtida apenas pelo exame clínico. No entanto, o tap test não pode ser usado como um teste de exclusão por causa da sua baixa sensibilidade (26\%-61\%). ${ }^{12,14,24}$

- Testes de resistência de saída de LCR (teste de infusão): são vários métodos para avaliar a dinâmica liquórica, geralmente envolvendo a infusão de soro fisiológico, Ringer lactato, ou CSF artificial no espaço subaracnóideo. Quando ocorre um estado de equilíbrio entre o volume infundido e o volume absorvido, a pressão é mensurada. Esse teste apresenta maior sensibilidade (57\%$100 \%$ ) em comparação com o tap test e um valor preditivo positivo de $75 \%$ a $92 \%$, ${ }^{6,12,24,26}$

- Drenagem lombar externa (DLE): é realizada drenagem contínua de LCR, geralmente em uma taxa de 5 a $10 \mathrm{ml}$ por hora, com a observação da resposta por mais de dois a sete dias no hospital. Uma DLE acima de $300 \mathrm{ml}$ está associada com alta sensibilidade (50\%-100\%) e alto valor preditivo positivo (80\%-100\%). Apesar de ser o teste suplementar que possui maior acurácia, também é o que possui maior taxa de complicação, além de requerer internação hospitalar. ${ }^{7,12,26}$

- Monitorização contínua da PIC: pode ser considerada durante a fase de diagnóstico/prognóstico da HPNi por meio da mensuração tanto da PIC estática (ondas B de Lundberg) quanto pulsátil. Valores de PIC elevados devem levar a uma reavaliação para descartar uma causa secundária de HPN. ${ }^{12,24}$ Eide e Sorteberg ${ }^{17}$ avaliaram a PIC estática e pulsátil de 214 pacientes portadores de HPNi. Desses, 131 foram submetidos à implantação de um sistema de derivação liquórica e 101 (93\%) apresentaram boa resposta após a cirurgia. Observou-se que em 109 pacientes as ondas médias da PIC pulsátil foram superiores a $4 \mathrm{mmHg}$. Dos 21 pacientes cujas ondas médias da PIC foram inferiores a $4 \mathrm{mmHg}$, apenas 2 responderam favoravelmente à cirurgia, sugerindo relação positiva entre a PIC pulsátil e a melhora após a derivação liquórica, no entanto esse exame não é comumente utilizado, visto se tratar de um procedimento invasivo.

- Avaliação do volume de ejeção liquórica no aqueduto do mesencéfalo (aqueduct stroke volume): como mencionado brevemente acima, o aqueduct stroke volume é definido como o volume médio de LCR passando através do aqueduto 
do mesencéfalo em uma direção crânio-caudal durante a sístole cardíaca e em uma direção caudocranial durante a diástole cardíaca, durante um único ciclo cardíaco, geralmente avaliado por meio de RM com as técnicas de cine-RM e phase contrast (PC). ${ }^{6,7,12,26,27}$ Scollato et al. ${ }^{27}$, em estudo que avaliou o volume de ejeção de LCR no aqueduto de 65 pacientes com HPN antes e após a implantação de DVP, observaram que nos 35 pacientes que melhoraram após a cirurgia, sua média pré-operatória de $157,01 \mu$ diminuiu para $18 \%$ um mês após a DVP e 49\% 12 meses após a cirurgia. Em um grupo de 15 pacientes que não melhoraram, a menor média do volume de ejeção no aqueduto observada no pré-operatório $(84,2$ $\mathrm{l}$ ) diminuiu para $14,3 \%$ um mês após a DVP e $34 \% 12$ meses após a DVP, sugerindo uma relação positiva entre melhora clínica e maiores quedas na taxa de volume de ejeção aquedutal após a DVP. Bateman e Loiselle ${ }^{3}$ avaliaram o volume de ejeção aquedutal em 32 pacientes submetidos à derivação liquórica (antes e depois da cirurgia). Desses, $63 \%$ apresentaram melhora após a derivação, no entanto, em relação ao volume de ejeção aquedutal, não se observou diferença entre os responsivos e os irresponsivos, contraindicando, dessa forma, a utilização desse exame como preditor de resposta após a cirurgia. A figura 8 apresenta uma sequência de etapas, tanto referentes à avaliação clínica, quanto relacionadas aos exames suplementares descritos acima, a fim de identificar quais pacientes mais provavelmente serão beneficiados após a implantação de um sistema de derivação liquórica. Tal algoritmo foi publicado na diretriz sobre HPNi de 2005 e inclui o grau de certeza de responsividade à derivação liquórica ofertado por cada procedimento. ${ }^{16}$

\section{Tratamento}

O tratamento da HPN visa, principalmente, restaurar a capacidade funcional do paciente. Dessa forma, a decisão sobre quando uma intervenção cirúrgica deve ou não ser realizada requer que se leve em consideração a probabilidade de tal restauração, por meio da utilização de algumas ferramentas preditoras do resultado pós-cirúrgico. Além dos testes suplementares abordados anteriormente, existem outros indicadores (favoráveis e desfavoráveis) que podem ser utilizados. Os indicadores favoráveis de melhora pós-cirúrgica incluem: início precoce do distúrbio de marcha (sendo o distúrbio de marcha a característica clínica mais proeminente); início dos sintomas de período inferior à seis meses. Os indicadores desfavoráveis incluem: ausência de distúrbio de marcha ou o seu surgimento após o início da demência; início precoce de demência; demência moderada a grave; presença de demência por mais de dois anos; atrofia difusa e importante comprometimento da substância branca no exame de RM..$^{10,29,30}$

A implantação de um sistema de derivação liquórica mediante intervenção cirúrgica é a medida terapêutica mais utilizada para a HPN. É realizada para aliviar o excesso de LCR no interior do sistema ventricular e tem demonstrado importantes benefícios. ${ }^{1,2,7,8,16-19,21,31-35}$ Diferentes tipos de derivação liquórica são utilizados, porém o mais comum é a DVP, mediante a utilização de um cateter delgado, cujo fluxo interior é unidirecional (craniocaudal), por causa da presença de um dispositivo valvulado acoplado ao sistema, que comunica os ventrículos cerebrais com a cavidade peritoneal, onde o excesso de LCR é drenado. ${ }^{21,28}$ A eficácia da DVP varia entre $33 \%$ e $90 \% .{ }^{31,35,36}$ Essa grande disparidade ocorre por causa da variação na seleção dos pacientes em diferentes estudos e do fato de não existir uma escala única para a análise de melhora do paciente que seja aceita universalmente. ${ }^{25} \mathrm{~A}$ taxa de complicações significantes (hemorragia intraoperatória grave, hematomas subdurais, déficits neurológicos, epilepsia, arritmias cardíacas, disfunção hipotalâmicas, fístulas liquóricas, infecções) ocorre em cerca de $6 \%$ dos pacientes após a cirurgia. ${ }^{12,19}$

Pujari et al..$^{20}$ analisaram retrospectivamente $55 \mathrm{pa}$ cientes acompanhados por pelo menos três anos após a cirurgia de DVP e relataram que em $53 \%$ desses pacientes foram necessárias revisões cirúrgicas. No entanto, observaram que, mesmo diante da necessidade de revisões, os pacientes em geral mantiveram melhora clínica em longo prazo que variou de cinco a sete anos, sobretudo relacionada ao distúrbio de marcha e incontinência urinária.

Kahlon et al. ${ }^{34}$ realizaram um estudo prospectivo de pacientes com suspeita de HPN, submetidos à DVP, seguidos em seis meses (54 pacientes) e cinco anos (23 pacientes). Relataram que em curto prazo (seis meses) $83 \%$ dos 54 pacientes operados melhoraram a marcha, $65 \%$ melhoraram o tempo de reação, $46 \%$ melhoraram nos testes de memória e $96 \%$ relataram melhora subjetiva. Em longo prazo (cinco anos) os 23 pacientes apresentaram melhora após a derivação de aproximadamente $40 \%$ na marcha, contudo menos de $10 \%$ na cognição e no tempo de reação. Os pacientes com menos de 75 anos tiveram uma taxa de melhora maior (64\%) que os com mais de 75 anos (11\%). Os autores afirmam que os benefícios da DVP podem ser obtidos até cinco anos após a cirurgia e fatores como taxa de mortalidade, idade e comorbidades devem ser levados em conta durante a seleção dos pacientes, visto que tais fatores alteram de maneira importante os resultados em curto e longo prazo. 


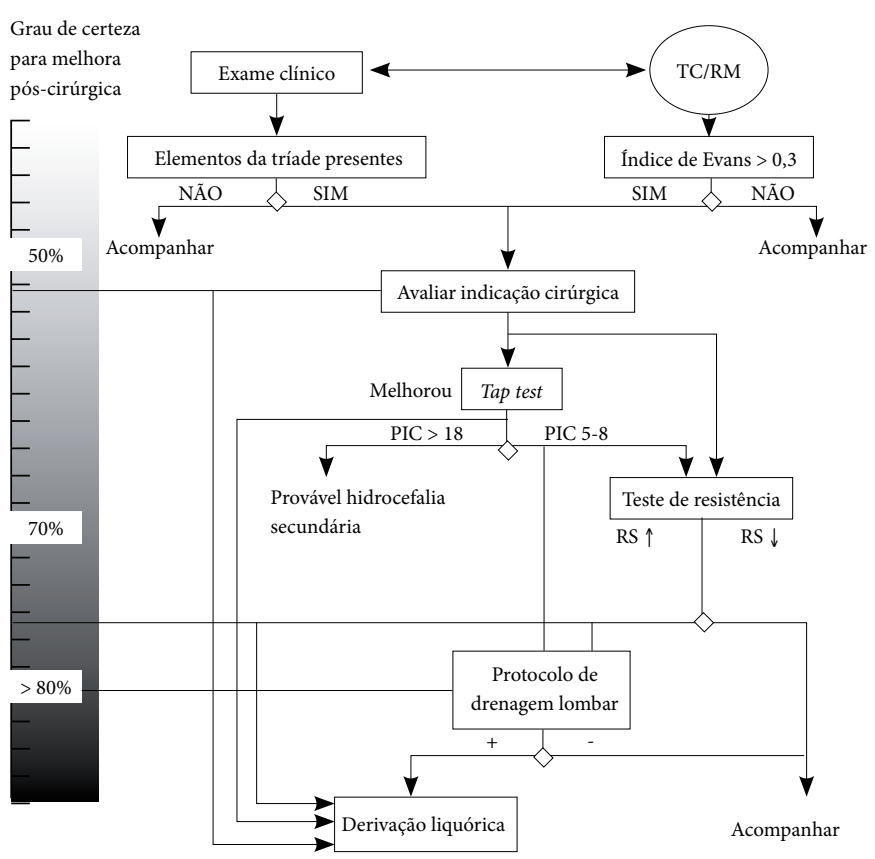

Figura 8 - Esquema para predizer a responsividade à derivação liquórica. A avaliação da indicação cirúrgica utilizando apenas os dados da avaliação clínica resultará em uma sensibilidade de $46 \%$ a $61 \%$ (escala à esquerda) em relação à melhora pós-cirúrgica. A resposta positiva ao tap test com retirada de 40 a $50 \mathrm{ml}$ de LCR é altamente preditiva de uma resposta favorável à derivação liquórica (72\%-100\%), porém sua sensibilidade é baixa (26\%-61\%). A mensuração da pressão de abertura do LCR durante o tap test é útil para que se possa excluir hidrocefalia secundária (PIC $>18 \mathrm{mmHg}$ ). O teste de resistência de saída do LCR (mediante sistema de infusão) fornece alta sensibilidade, variando de $57 \%$ a $100 \%$, e está associado a um valor preditivo positivo de $75 \%$ a $92 \%$. A drenagem lombar externa é o teste suplementar que fornece a maior sensibilidade (50\%-100\%) e o maior valor preditivo positivo (80\%-100\%), no entanto requer internação hospitalar e é o que mais está associado a complicações. RS: resistência de saída do LCR. Esse esquema foi publicado no artigo: Marmarou A, et al. The value of supplemental 'prognostic tests for the preoperative assessment of idiopathic normal pressure hydrocephalus'. Neurosurgery. 2005;57(Suppl 3):17-28. ${ }^{26}$ (Traduzido)

A terceira ventriculostomia endoscópica (TVE) também tem sido utilizada no tratamento da HPN., ${ }^{8,16,32}$ Considerada uma derivação interna, tal técnica consiste na fenestração do assoalho do terceiro ventrículo, permitindo que o LCR passe diretamente do terceiro ventrículo para o compartimento anterior da cisterna interpeduncular, aumentando o fluxo sistólico de saída dos ventrículos (o que resulta em diminuição do excesso de LCR intraventricular) e diminuindo os efeitos deletérios do LCR sobre as paredes ventriculares durante as ondas sistólicas pulsáteis. Gangemi et al. ${ }^{8}$ avaliaram retrospectivamente 110 pacientes com HPN submetidos à TVE. Observou-se que ocorreu melhora clínica pós-operatória em $76(69,1 \%)$ pacientes. Não houve correlação entre a taxa de sucesso e a idade dos pacientes ou tipo de aumento ventricular. Entretanto, a taxa de melhora neurológica foi maior em pacientes com menor tempo de história clínica e distúrbios de marcha no início da doença. No intraoperatório, o reaparecimento das pulsações cerebrais normais e a observação de movimentos significantes do assoalho do terceiro ventrículo, para cima e para baixo, após a TVE, foram relacionados com um bom resultado. Complicações ocorreram em $7(6,4 \%)$ pacientes e a necessidade de um segundo procedimento endoscópico em $4(3,6 \%)$ pacientes. Portanto, os autores concluem que a TVE é uma opção eficaz e segura para o tratamento da HPN.

Outros exemplos de derivação que raramente são usados incluem a derivação ventrículo-atrial e a DLP. ${ }^{6,12,17}$

\section{Conclusão}

A HPN é uma doença insidiosa que acomete, principalmente, idosos. É uma das poucas causas reversíveis de demência, respondendo por cerca de $5 \%$ dos casos. As hipóteses referentes à sua fisiopatologia baseiam-se, sobretudo, nas alterações da hemodinâmica cerebral e dinâmica liquórica. É fundamental que o diagnóstico da HPN seja realizado precocemente, por meio do conhecimento sobre a doença e seus diferenciais, por parte dos clínicos, geriatras e neurologistas, e que os pacientes com boas chances de responder ao tratamento sejam identificados mediante a utilização dos exames preditores de eficácia disponíveis. Mais estudos são necessários para que critérios diagnósticos sejam padronizados e métodos universais de avaliação pós-cirúrgica sejam estabelecidos, a fim de definir quais métodos terapêuticos são mais eficazes e seguros no manejo cirúrgico da HPN. 


\section{Referências}

1. Hakim S. Some observations on CSF pressure: hydrocephalic syndrome in adults with "normal" CSF pressure [tese]. Bogotá: Universidade Javeriana da Faculdade de Medicina; 1964.

2. Hakim S, Adams RD. The special clinical problem of symptomatic hydrocephalus with normal cerebrospinal fluid pressure: observations on cerebrospinal fluid hydrodynamics. J Neurol Sci. 1965;2(4):307-27.

3. Bateman GA, Loiselle AM. Can MR measurement of intracranial hydrodynamics and compliance differentiate which patient with idiopathic normal pressure hydrocephalus will improve following shunt insertion?. Acta Neurochir (Wien). 2007;149(5):455-62.

4. Wallenstein MB, McKhann GM 2nd. Salomón Hakim and the discovery of normal-pressure hydrocephalus. Neurosurgery. 2010;67(1):155-9.

5. Brean A, Eide PK. Prevalence of probable idiopathic normal pressure hydrocephalus in a Norwegian population. Acta Neurol Scand. 2008;118(1):48-53.

6. Chen YF, Wang YH, Hsiao JK, Lai DM, Liao CC, Tu YK, et al. Normal pressure hydrocephalus: cerebral hemodynamic, metabolism measurement, discharge score, and long-term outcome. Surg Neurol. 2008;70(Suppl 1):S1:69-77.

7. Eide PK. Intracranial pressure parameters in idiopathic normal pressure hydrocephalus patients treated with ventriculo-peritoneal shunts. Acta Neurochir (Wien). 2006;148(1):21-9.

8. Gangemi M, Maiuri F, Naddeo M, Godano U, Mascari C, Broggi $G$, et al. Endoscopic third ventriculostomy in idiopathic normal pressure hydrocephalus: an Italian multicenter study. Neurosurgery. 2008;63(1):62-7.

9. Marmarou A, Young HF, Aygok GA, Sawauchi S, Tsuji O, Yamamoto T, et al. Diagnosis and management of idiopathic normal-pressure hydrocephalus: a prospective study in 151 patients. J Neurosurg. 2005;102(6):987-97.

10. Mori K. Management of idiopathic normal-pressure hydrocephalus: A multiinstitutional study conducted in Japan. J Neurosurg. 2001;95(6):970-3.

11. Takeuchi T, Goto H, Izaki K, Tamura S, Tomii M, Sasanuma $\mathrm{J}$, et al. Pathophysiology of cerebral circulatory disorders in idiopathic normal pressure hydrocephalus. Neurol Med Chir (Tokyo). 2007;47(7):299-306.

12. Chang CC, Asada H, Mimura T, Suzuki S. A prospective study of cerebral blood flow and cerebrovascular reactivity to acetazolamide in 162 patients with idiopathic normalpressure hydrocephalus. J Neurosurg. 2009;111(3):610-7.

13. Chrysikopoulos H. Idiopathic normal pressure hydrocephalus: thoughts on etiology and pathophysiology. Med Hypotheses. 2009;73(5):718-24.

14. Shprecher D, Schwalb J, Kurlan R. Normal pressure hydrocephalus: diagnosis and treatment. Curr Neurol Neurosci Rep. 2008;8(5):371-6.

15. Relkin N, Marmarou A, Klinge P, Bergsneider M, Black PM. Diagnosing idiopathic normal-pressure hydrocephalus. Neurosurgery. 2005;57(3 Suppl):S4-16.

16. Fabiano AJ, Doyle K, Grand W. Delayed stoma failure in adult communicating hydrocephalus after initial successful treatment by endoscopic third ventriculostomy: case report. Neurosurgery. 2010;66(6):E1210-1.

17. Eide $\mathrm{PK}$, Sorteberg W. Diagnostic intracranial pressure monitoring and surgical management in idiopathic normal pressure hydrocephalus: a 6-year review of 214 patients. Neurosurgery. 2010;66(1):80-91.

18. Factora R. When do common symptoms indicate normal pressure hydrocephalus? Cleve Clin J Med. 2006;73(5):447$50,452,455-6$.
19. Klinge $\mathrm{P}$, Marmarou A, Bergsneider M, Relkin N, Black $\mathrm{PM}$. Outcome of shunting in idiopathic normal-pressure hydrocephalus and the value of outcome assessment in shunted patients. Neurosurgery. 2005;57(3 Suppl):S40-52.

20. Pujari S, Kharkar S, Metellus P, Shuck J, Williams MA, Rigamonti D. Normal pressure hydrocephalus: long-term outcome after shunt surgery. J Neurol Neurosurg Psychiatry. 2008;79(11):1282-6.

21. Gallia GL, Rigamonti D, Williams MA. The diagnosis and treatment of idiopathic normal pressure hydrocephalus. Nat Clin Pract Neurol. 2006;2(7):375-81.

22. Sakakibara R, Kanda T, Sekido T, Uchiyama T, Awa $\mathrm{Y}$, Ito $\mathrm{T}$, et al. Mechanism of bladder dysfunction in idiopathic normal pressure hydrocephalus. Neurourol Urodyn. 2008;27(6):507-10.

23. Wilson RK, Williams MA. The role of the neurologist in the longitudinal management of normal pressure hydrocephalus. Neurologist. 2010;16(4):238-48.

24. Bateman GA. The pathophysiology of idiopathic normal pressure hydrocephalus: cerebral ischemia or altered venous hemodynamics? AJNR Am J Neuroradiol. 2008;29(1):198-203.

25. Takaya M, Kazui H, Tokunaga H, Yoshida T, Kito Y, Wada $\mathrm{T}$, et al. Global cerebral hypoperfusion in preclinical stage of idiopathic normal pressure hydrocephalus. J Neurol Sci. 2010(15);298(1-2):35-41.

26. Marmarou A, Bergsneider M, Klinge P, Relkin N, Black $\mathrm{PM}$. The value of supplemental prognostic tests for the preoperative assessment of idiopathic normal-pressure hydrocephalus. Neurosurgery. 2005;57(3 Suppl):S17-28.

27. Scollato A, Gallina P, Gautam B, Pellicanò G, Cavallini C, Tenenbaum R, et al. Changes in aqueductal CSF stroke volume in shunted patients with idiopathic normal-pressure hydrocephalus. AJNR Am J Neuroradiol. 2009;30(8):1580-6.

28. Siraj S. An overview of normal pressure hydrocephalus and its importance: how much do we really know? J Am Med Dir Assoc. 2011;12(1):19-21.

29. Black PM, Ojemann RG, Tzouras A. CSF shunts for dementia, incontinence, and gait disturbance. J Clin Neurosurg. 1985;32:632-51.

30. Petersen RC, Mokri B, Laws ER Jr. Surgical treatment of idiopathic hydrocephalus in elderly patients. Neurology. 1985;35(3):307-11.

31. Gleason PL, Black PM, Matsumae M. The neurobiology of normal pressure hydrocephalus. J Neurosurg Clin N Am. 1993;4(4):667-75.

32. Hailong F, Guangfu H, Haibin T, Hong P, Yong C, Weidong L, et al. Endoscopic third ventriculostomy in the management of communicating hydrocephalus: a preliminary study. $J$ Neurosurg. 2008;109(5):923-30.

33. Hebb AO, Cusimano MD. Idiopathic normal pressure hydrocephalus: A systematic review of diagnosis and outcome. J Neurosurg. 2001;49(5):1166-84.

34. Kahlon B, Sjunnesson J, Rehncrona S. Long-term outcome in patients with suspected normal pressure hydrocephalus. Neurosurgery. 2007;60(2):327-32.

35. Krauss JK, Faist M, Schubert M, Borremans JJ, Lücking $\mathrm{CH}$, Berger W. Evaluation of gait in normal pressure hydrocephalus before and after shunting. J Adv Neurol 2001;87:301-10.

36. Verrees M, Selman WR. Management of normal pressure hydrocephalus. Am Fam Physician. 2004;70(6):1071-8.

\section{Endereço para correspondência}

Renan Muralho Pereira

Rua Cravo da Índia, 599, Pq. Savoy City 03570-050 - São Paulo, SP

Telefones: (11) 8227-2414/(11) 7623-5730

E-mail: renanfp@msn.com 


\title{
Morte encefálica: diagnóstico possível sem utilização de exames complementares
}

\author{
Fernanda de Paula Pimenta1, Bárbara Rios Vellasco Amorim¹, Ledismar José Silva² \\ Hospital de Urgências de Goiânia Dr. Valdemiro Cruz (HUGO) e Hospital Santa Mônica, Goiânia, Goiás.
}

\section{RESUMO}

Objetivo: A necessidade da utilização de testes confirmatórios para o diagnóstico de morte encefálica vem sendo questionada nos últimos tempos. Acredita-se que o exame clínico completo, realizado de acordo com o protocolo do Conselho Federal de Medicina, seja suficiente para constatar a ausência irreversível de função no tronco encefálico. Assim, este estudo teve como objetivo averiguar a real necessidade da utilização de exames complementares no diagnóstico de morte encefálica. Método: Foi realizado estudo prospectivo analisando os prontuários de pacientes que receberam diagnóstico de morte encefálica desde a abertura do protocolo até a realização dos exames clínicos completos e exame complementar (Doppler transcraniano). Resultados: Não foi registrado nenhum caso de recuperação da função cortical elou do tronco cerebral após o diagnóstico de morte encefálica utilizando os critérios clínicos. A dificuldade para a realização do Doppler transcraniano e as falhas inerentes ao exame contribuíram para tornar mais lento o diagnóstico. Conclusão: É possível diagnosticar morte encefálica apenas utilizando exame clínico completo, sem a necessidade de exames complementares.

\section{PALAVRAS-CHAVE}

Morte encefálica, diagnóstico clínico, estudos prospectivos.

\begin{abstract}
Brain death: possible diagnosis without complementary exams

Objective: The need to use confirmatory tests for brain death diagnosis has been questioned lately. It is believed that a full clinical examination, performed according to the protocol of brain death diagnosis of the Brazilian National Council of Medicine, is sufficient to certify the irreversible loss of brain-stem function. Therefore, this study aimed to verify the real need to use complementary exams for brain death diagnosis. Method: We carried out a prospective study analyzing the files of patients with brain death diagnoses since the beginning of the protocol and that underwent a full clinical examination and transcranial Doppler as a complementary exam. Results: No cases of cortical and/or brain-stem function recovery after brain death diagnosis using clinical criteria were registered. Difficulty to perform transcranial Doppler and failures inherent to the exam contributed to late diagnosis. Conclusion: It is possible to diagnose brain death only using a full clinical examination, with no need to perform complementary exams.
\end{abstract}

\section{KEYWORDS}

Brain death, clinical diagnosis, prospective studies.

\section{Introdução}

O conceito de morte vem sofrendo mudanças no decorrer do tempo. Os avanços da medicina, que incluem técnicas de ressuscitação cardíaca, respiradores artificiais e circulação extracorpórea, tornaram obsoleta a definição tradicional de morte clínica, que incluía apenas a cessação irreversível das funções cardíaca e respiratória. ${ }^{1} \mathrm{O}$ novo conceito de morte, formulado em 1981 pela President's Commission for the Study of Ethical Problems in Medicine and Biomedical and Behavioral Research, dos Estados Unidos, amplamente aceito até hoje, define-a como a cessação irreversível das funções cardíacas, respiratórias e de todo o encéfalo, em especial o tronco encefálico. ${ }^{1,2}$

Os critérios para diagnóstico de morte encefálica (ME) no Brasil são baseados na constatação clínica

1. Acadêmica de Medicina da Pontifícia Universidade Católica de Goiás (PUC-GO), Goiânia, GO, Brasil.

2. Neurocirurgião e docente do Departamento de Medicina da PUC-GO, Goiânia, GO, Brasil. 